\title{
Supplementary information to Identifying global patterns of stochasticity and nonlinearity in the Earth System
}

\author{
Fernando Arizmendi ${ }^{\dagger}$, Marcelo Barreiro ${ }^{\dagger}$, and Cristina Masoller ${ }^{\S *}$ \\ ${ }^{\dagger}$ Instituto de Física, Facultad de Ciencias, Universidad de la República, Iguá 4225, Montevideo, Uruguay and \\ ${ }^{\S}$ Departament de Física, Universitat Politecnica de Catalunya, 08222 Terrassa, Barcelona, Spain
}

(Dated: March 30, 2016)

\begin{abstract}
We present a detailed analysis of the measure proposed in the main text to assess atmospheric response to solar forcing: we analyse the role of the interval in which we search for the minimum distance between time series, and we compare with an alternative measure. We also present a comparison of the results obtained from the two databases (NCEP CDAS1 and ERA Interim).
\end{abstract}

In the main text we search for the minimum of the difference between two time-series, $x_{i}(t)$ and $y_{i}(t)$, Eq. (1) (reproduced here for convenience),

$$
d_{i}=\sum_{t=1}^{L}\left|y_{i}(t)-x_{i}\left(t+\tau_{i}\right)\right|
$$

with $\tau_{i}$ in the interval $[0,4]$. In Fig. 1 we discuss the influence of searching the minimum in the intervals $[0,2]$, $[0,3]$ and $[0,5]$. We observe that similar spatial patterns are obtained, confirming the robustness of the method- ology.

As discussed in the main text, if instead of using $\left|y_{i}(t)-x_{i}\left(t+\tau_{i}\right)\right|$ to define the difference, we use $\left(y_{i}(t)-\right.$ $\left.x_{i}\left(t+\tau_{i}\right)\right)^{2}$, then $d_{i}$ is equal to $2\left(1-\rho_{i}\right)$ where $\rho_{i}$ is the cross-correlation coefficient between $y_{i}(t)$ and $x_{i}\left(t+\tau_{i}\right)$. In Fig. 2 we compare the two approaches, and again we obtain very similar spatial patterns.

Figures 3 and 4 display the comparison of the results obtained from the two databases (NCEP CDAS1 and ERA Interim) and we observe a good qualitative agreement, except for well-localized regions, where extreme values occur as discussed in the main text. 

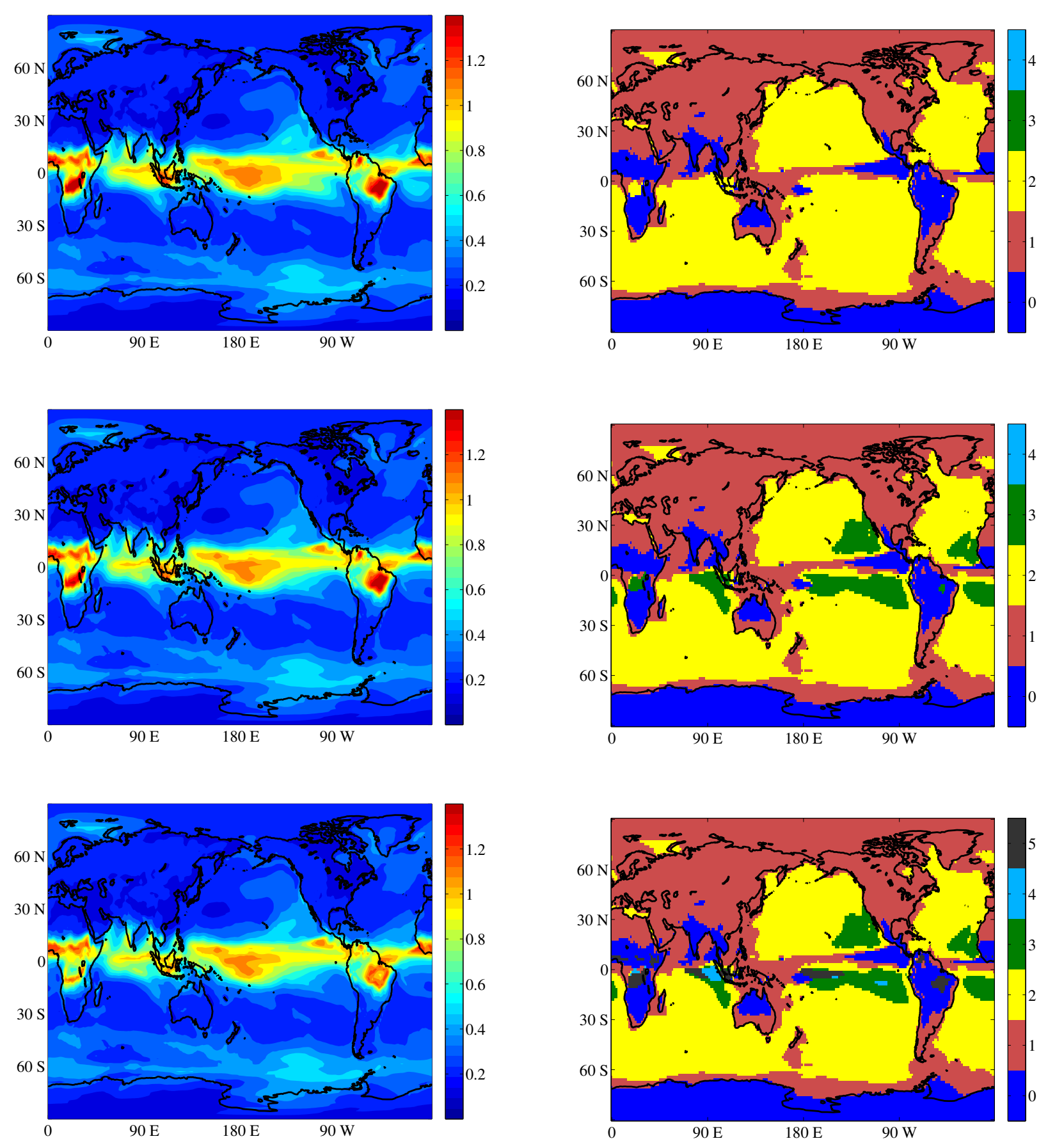

FIG. 1. Maps of distances $d_{i}$ (left) calculated from Eq. (1) when the forcing and the response are shifted $\tau_{i} \in[0,2]$ (a), $\tau_{i} \in[0,3]$ (b) and $\tau_{i} \in[0,5]$ (c). Maps of respective lags $\tau_{i}$ (left). 

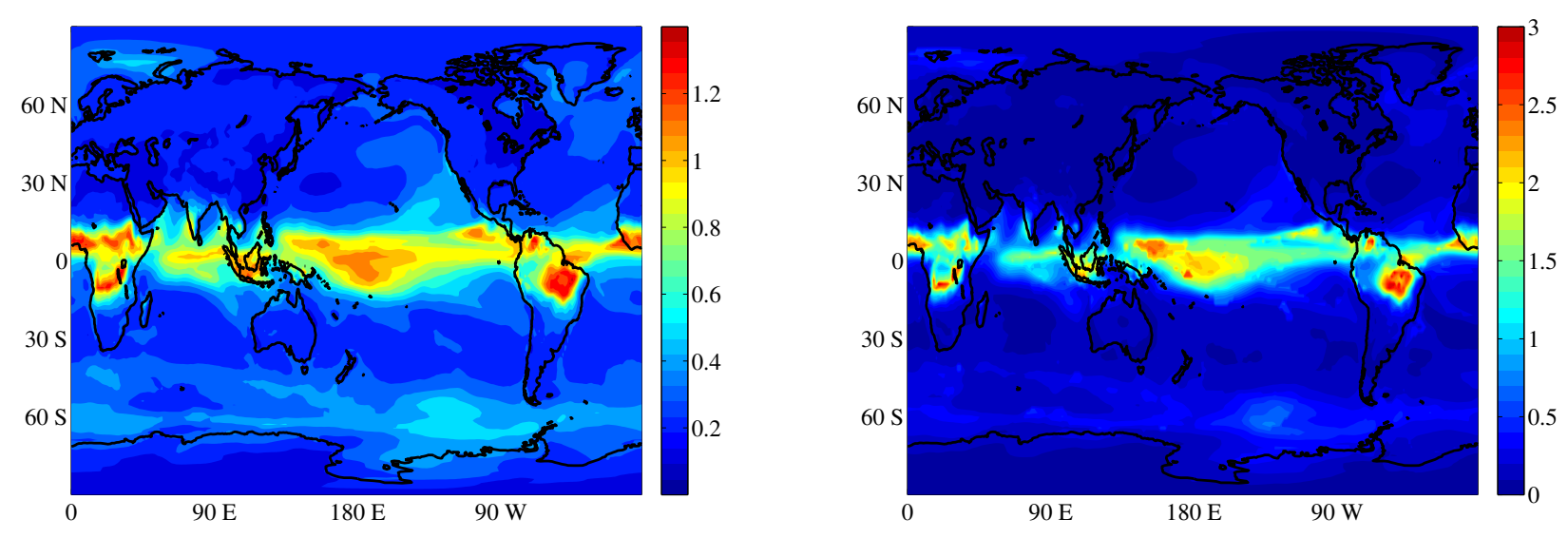

FIG. 2. Map of distances $d_{i}$ calculated from Eq. (1) (a), and when is calculated from Eq.(1), but replacing $\left|y_{i}(t)-x_{i}\left(t+\tau_{i}\right)\right|$ by $\left(y_{i}(t)-x_{i}\left(t+\tau_{i}\right)\right)^{2}$. In both cases, the forcing and the response are shifted $\tau_{i}$, with $\tau_{i} \in[0,4]$.
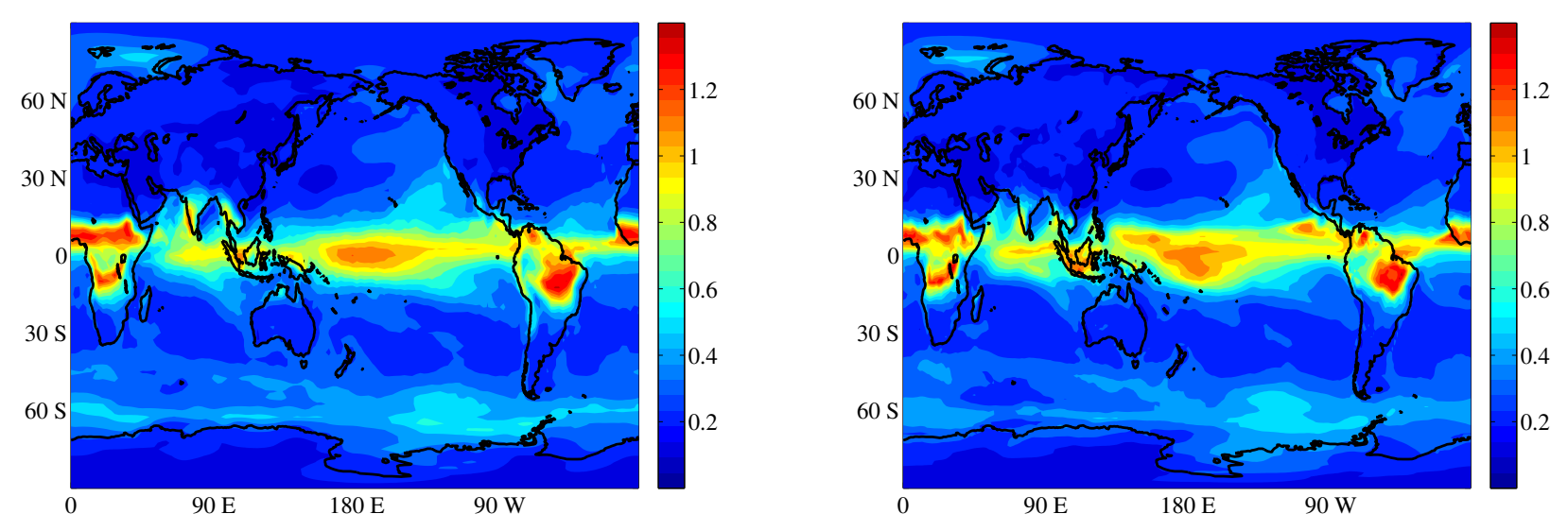

FIG. 3. Map of distances $d_{i}$ calculated from Eq. (1) with $\tau_{i} \in[0,4]$ obtained from NCEP CDAS1 (left) and ERA Interim (right).
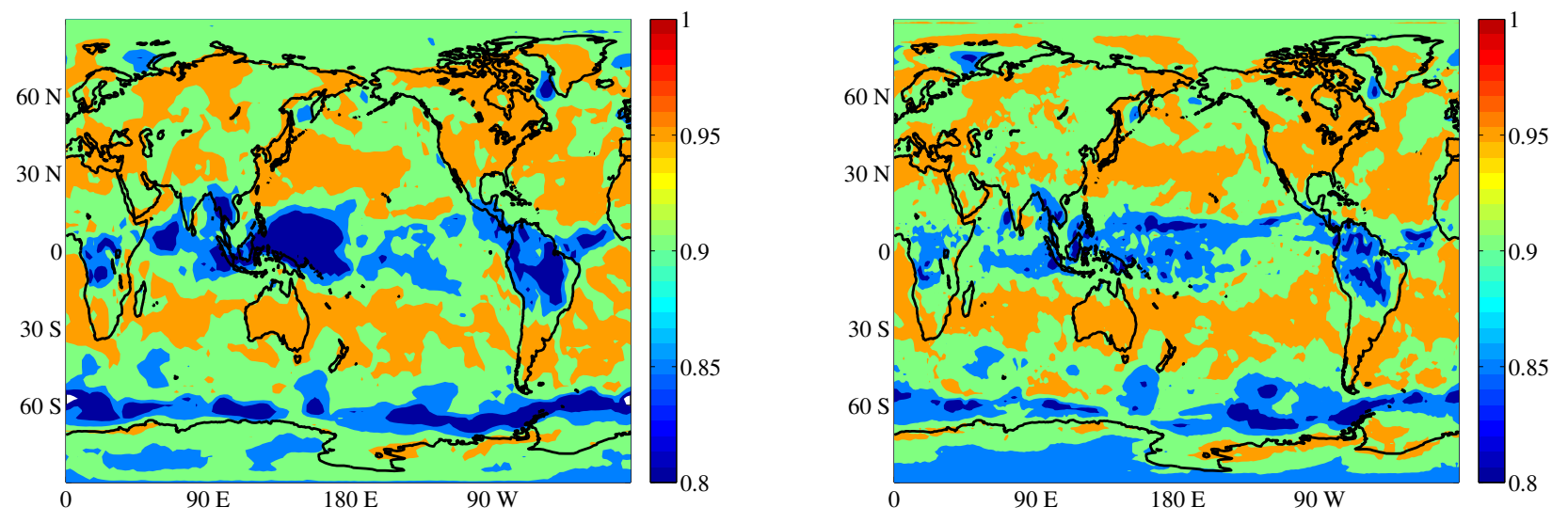

FIG. 4. Entropy maps obtained from NCEP CDAS1 (left) and ERA Interim (right), computed from SAT time-series. 